\title{
Xingnaojing for Moderate-to-severe Acute ischemic Stroke (XMAS): study protocol for a randomized controlled trial
}

\author{
Xinxing Lai', Kegang Cao ${ }^{1,2}$, Lingbo Kong ${ }^{2,3}$, Qiang Liu ${ }^{4}$, Ying Gao ${ }^{1,2^{*}}$ (D) and for the XMAS study investigators
}

\begin{abstract}
Background: Xingnaojing injection (XNJ) is widely used for the treatment of stroke in China. However, there is currently a lack of high-quality evidence of its efficacy for acute ischemic stroke. The main objective of this study is to determine whether the addition of XNJ to standard care improves the 3-month functional outcome in patients with acute ischemic stroke (AIS).

Methods/design: The XMAS study is a multicenter, prospective, randomized controlled, open-label trial with a blinded endpoints design. A total of 720 patients will be randomly allocated to either the intervention or the control group in a 1:1 ratio. The intervention group receives XNJ combined with standard care, and the control group receives standard care alone. XNJ will be administered intravenously every $12 \mathrm{~h}$ for 10 days. The primary outcome is the proportion of patients who are independent at 3 months after stroke onset defined as a modified Rankin Scale score of 0 to 2. Secondary outcomes include early neurological deterioration at $48 \mathrm{~h}$, the change in National Institutes of Health Stroke Scale score, patient-reported outcome, symptomatic intracranial hemorrhage at 10 days, the Barthel Index score, deaths from any cause and cardiovascular events at 3 months.
\end{abstract}

Discussion: The results of this trial will provide critical evidence for XNJ in the treatment of AIS as a complementary approach that can be initiated after reperfusion therapy or when the AIS is not eligible for thrombolytic treatment.

Trial registration: Clinical Trials.gov, ID: NCT02728180. Registered on 28 March 2016.

Keywords: Xingnaojing injection, Chinese medicine, Acute ischemic stroke, Randomized controlled trial

\section{Background}

Globally, stroke is one of the leading causes of death and long-term disability [1]. In the past two decades, the burden of stroke has increased significantly in terms of the absolute number of people with incident stroke, number of deaths, and disability-adjusted life years (DALYs) lost especially in developing countries [2]. According to a nationwide population-based survey in China, there are approximately 2.4 million new strokes and 1.1 million stroke-related deaths annually, with

\footnotetext{
* Correspondence: gaoying973@163.com

'Department of Neurology, Dongzhimen Hospital affiliated to Beijing University of Chinese Medicine, 5 Haiyuncang, Dongcheng District, Beijing 100029, China

${ }^{2}$ Institute for Cerebrovascular Disease of Beijing University of Chinese Medicine, 5 Haiyuncang, Dongcheng District, Beijing 100029, China Full list of author information is available at the end of the article
}

11.1 million stroke survivors, which consequently results in a huge financial burden [3].

Despite recombinant tissue plasminogen activator (rt-PA) and emerging evidence for endovascular treatment [4], only a few highly selected patients with acute ischemic stroke (AIS) receive thrombolytic therapy due to strict selection criteria. From the Chinese National Stroke Registry, $21.5 \%$ of patients with acute stroke presented to the emergency department (ED) within 3 hours, $12.6 \%$ were eligible for thrombolytic treatment, and only $1.6 \%$ received intravenously administered rt-PA therapy [5]. The lack of effective and widely applicable therapeutic approaches for AIS has resulted in a growing interest in traditional Chinese medicine (TCM).

Xingnaojing injection (XNJ) was extracted by modern biotechnology from a well-known Chinese patent 
medicine named An-Gong-Niu-Huang Wan which was first documented in Wen Bing Tiao Bian, a classical work of TCM published in 1798. XNJ has been approved by the China Food and Drug Administration (CFDA) and is widely used for the treatment of stroke [6]. XNJ consists of four Chinese herbs: Moschus, Radix curcumae, borneol, and Fructus gardeniae. Previous studies have demonstrated that XNJ may has multiple neuroprotective mechanisms in terms of inhibiting glutamate-induced apoptosis [7], anti-autophagy via the p53-DRAM signaling pathway [8], decreasing gamma-aminobutyric acid (GABA) [9], improving neurobehavioral disturbances, as well as reducing infarct size [10]. Recently, a systematic review and meta-analysis to assess the efficacy of XNJ for stroke demonstrated that XNJ may reduce brain injury and improve functional recovery after stroke. Due to methodological limitations of the included trials, however, the efficacy of $\mathrm{XNJ}$ for ischemic stroke is still not established [11].

Thus, in the present study, we aim to determine whether the addition of $\mathrm{XNJ}$ to standard care, intravenously administered within $24 \mathrm{~h}$ of symptom onset, improves the 3month functional outcome in participants with AIS.

\section{Methods/design}

\section{Study design}

The Xingnaojing for Moderate-to-severe Acute ischemic Stroke (XMAS) trial is a multicenter, prospective, randomized controlled, open-label trial with blinded endpoints assessment (PROBE design). We compare XNJ, in combination with guideline-based standard care, versus standard care alone in patients with acute ischemic stroke within $24 \mathrm{~h}$ after stroke onset. A completed Standard Protocol items: Recommendations for Interventional Trials (SPIRIT) Checklist for the trial is available in Additional file 1.

\section{Recruitment}

Patients will be recruited from 30 tertiary public hospitals throughout China with EDs and neurology wards that receive patients with AIS. The recruitment started in March 2016 and is estimated to end in January 2018. Patients with moderate-to-severe AIS within $24 \mathrm{~h}$ after stroke onset will be screened for eligibility according to the inclusion and exclusion criteria. Moderate-to-severe was defined as the National Institutes of Health Stroke Scale (NIHSS) scores ranging from 5 to 20. Time of stroke onset is defined as the last time when the patient was witnessed to be well. Those who have stroke symptoms upon awakening (wake-up stroke) will be considered to have their onset at beginning of sleep. Only those who meet the inclusion criteria and willingly provide written informed consent will be included. The flow diagram of this study is shown in Fig. 1.

\section{Inclusion criteria}

- Acute ischemic stroke within $24 \mathrm{~h}$ of symptom onset

- NIHSS score $\geq 5$ and $\leq 20$

- Age $\geq 35$ and $\leq 80$ years

- Patient or legally authorized representative has signed informed consent

\section{Exclusion criteria}

- Planned or already receiving endovascular treatment

- Suspected secondary stroke caused by tumor, brain trauma, or hematological diseases

- Already dependent in activities of daily living before the present acute stroke (defined as modified Rankin Scale score $\geq 2$ )

- Other conditions that lead to motor dysfunction (e.g., severe osteoarthrosis, rheumatoid arthritis)

- Significant renal or hepatic insufficiency (defined as a serum creatinine concentration, alanine aminotransferase (ALT), or aspartate aminotransferase (AST) value that is twice the upper limit of normal)

- Life expectancy of 3 months or less due to other life-threatening illness (e.g., advanced cancer)

- Other conditions that render outcomes or follow-up unlikely to be assessed

- Known to be pregnant or breastfeeding

- Currently receiving an investigational drug

\section{Randomization}

All participants will be randomly allocated to the intervention group or the control group in a 1:1 ratio. The randomization procedure will be computer- and webbased, using permuted blocks, and will be stratified for medical center. The randomization algorithm for the study will be generated and uploaded to the randomization system before recruitment. It will not be possible to know the treatment allocation before randomization. The passwordprotected randomization system requests a few key items of baseline data including the time of stroke onset, NIHSS score, date of birth, as well as a double check on inclusion and exclusion criteria, which are then entered with the computer keypad or tablet computer. When the data have been entered and checked, the computer will generate the treatment allocation. Participants who do not meet the criteria will be excluded by the system automatically and will not be randomized.

\section{Blinding}

In this PROBE design trial, both participants and treating clinicians will be aware of the treatment assignment. Information including NIHSS score, Glasgow Coma 


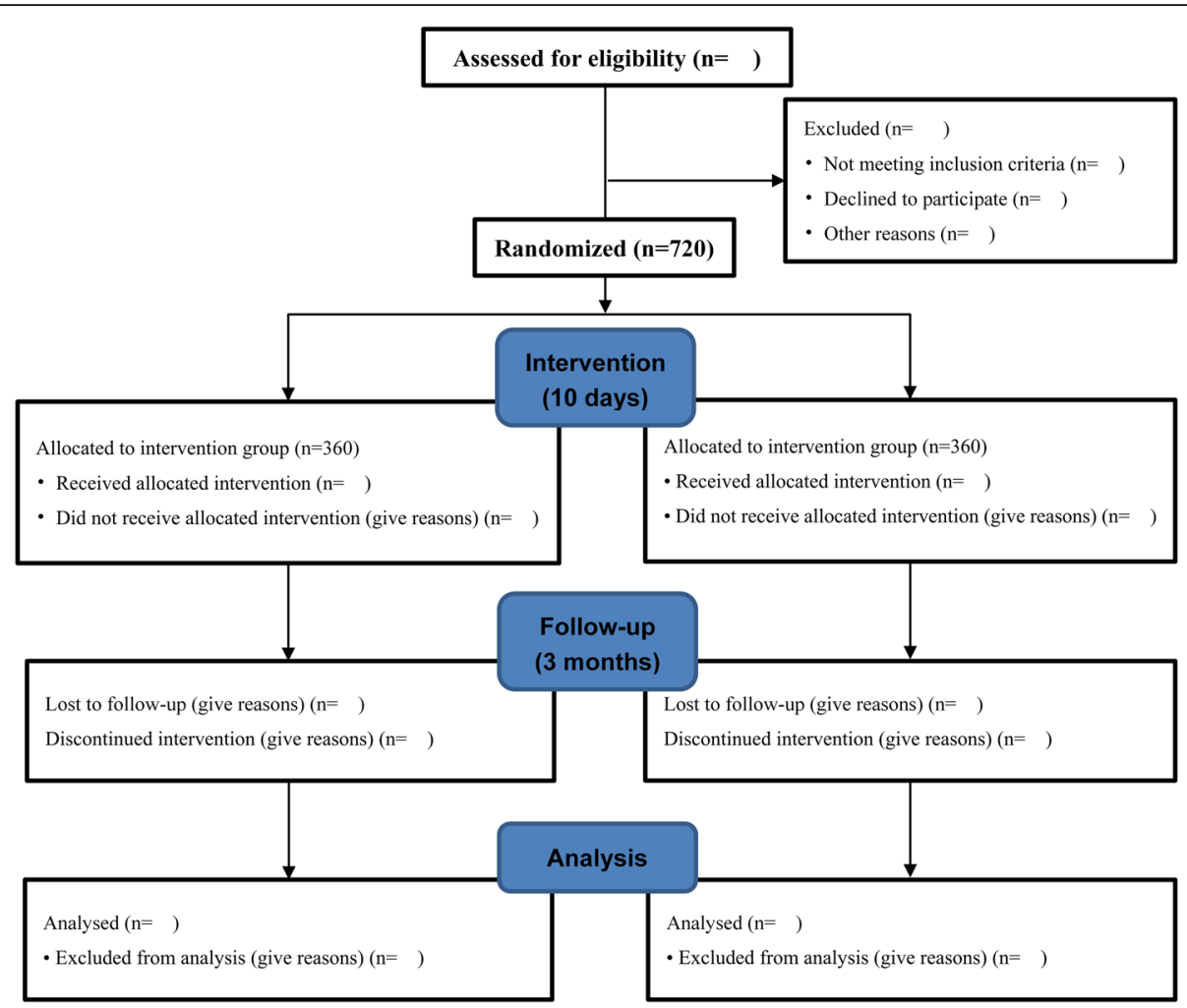

Fig. 1 Flow diagram of the Xingnaojing for Moderate-to-severe Acute ischemic Stroke (XMAS) study

Scale (GCS) score, and Patient-reported Outcome (PRO) score will be assessed by independent assessors who are blind to the assignment and treatment. Patients will be contacted over the telephone by independent trained interviewers, who will use standard structured scripts to collect planed data at 30 days and 3 months after stroke onset. The principal investigator, statistician, and outcomes assessors were unaware of the treatment assignments throughout the trial until the database was locked.

\section{Interventions}

Patients randomly assigned to the intervention group will receive $\mathrm{XNJ}$ injection, which will be manufactured by the Jiminkexin Group, combined with standard stroke care. XNJ will be administered intravenously in the ED or neurology ward immediately after completion of the randomization. The infusion will contain $20 \mathrm{ml} \mathrm{XNJ} \mathrm{di-}$ luted in $250 \mathrm{ml}$ normal saline, infused every $12 \mathrm{~h}$ for 10 days. All information regarding $\mathrm{XNJ}$ administered to each participant will be recorded by nurses including dosage, administration time, and details of discontinued treatment.

Those who are assigned to the control group will be given standard stroke care alone. The standard stroke care follows the current national guidelines for acute ischemic stroke from the Chinese Society of Neurology, including anti-platelet therapy, control of vascular risk factors, and appropriate rehabilitation, which will be determined by the attending physician. Treatment with tissue plasminogen activator (rt-PA) within 3 to $4.5 \mathrm{~h}$ of symptom onset, after exclusion of intracranial hemorrhage, is encouraged in participants who meet thrombolytic treatment criteria outlined in guidelines.

Neither traditional Chinese herbal medication nor acupuncture is allowed during the hospitalization. All concomitant medications will be recorded in the Case Report Forms (CRFs) including the name, dosage, and course of these medications.

Participants may withdraw at any time during the trial. Investigators will discontinue the treatment in the following cases: the occurrence of serious adverse events; at the request of participant or legally authorized representative to discontinue; and other reasons for consideration of safety. All reasons of discontinuing will be recorded in the CRFs.

\section{Outcome measures}

\section{Primary outcome}

The primary outcome is the proportion of patients independent at 3 months after stroke onset defined by a mRS score of 0,1 , or 2 . Scores on this scale range from 0 to 6 , with higher scores indicating greater disability [12]. 


\section{Secondary outcomes}

- Comparison of the change of degree of neurological deficit from baseline to day 10 or discharge, according to the NIHSS scores ranging from 0 to 42 , with higher scores indicating more severe neurological deficits [13]

- Comparison of the PRO scale of stroke at 10 days, which consists of four dimensions including the influence on physical, emotional, and social functioning, as well as the overall satisfaction with treatment [14]

- The proportion of patients with a Barthel Index (BI) score of $\geq 90$ at 30 days and 3 months after stroke onset. The $\mathrm{BI}$ is used to evaluate the activities of daily living, ranging from 0 to 100 , with higher scores indicating more independence [15]

- The proportion of early neurological deterioration (END), defined as an increase of 3 points or more in the NIHSS score between baseline and $48 \mathrm{~h}$

- The proportion of symptomatic intracranial hemorrhage (sICH) occurrence within 10 days of stroke onset, defined as any $\mathrm{ICH}$ related to a decline in neurological status or the development of new neurological symptoms in the judgment of the clinical investigator

- The incidence of cardiovascular events, which consist of recurrence of stroke or myocardial infarction

- The incidence of deaths from any cause within 10 days and 3 months after symptom onset

- Safety endpoints, which consist of any adverse events, results of electrocardiography, vital signs and laboratory tests (complete blood count, chemistry, and urinalysis)

\section{Safety reporting}

Adverse events (AEs) are defined as any unfavorable and unintended sign, symptom or disease arising in participants in both groups during the trial period, whether or not related to the experimental treatment. The investigator should take appropriate measures to ensure the safety of the participants and follow up the outcome of any AEs (clinical signs, laboratory values or other, etc.) until the return to normal or stabilization of the patient's condition. All AEs reported by the subjects, caregivers, or observed by the treating physicians during this trial will be recorded, including the nature of each event, onset date and time, duration, intensity, assessment of its cause, specific therapy and its outcome.

A serious adverse event (SAE) is one that can fatal or life-threatening, requires prolonged hospitalization, or results in permanent or substantial disability. In case of SAEs, the principal investigator will be notified by email or telephone within $24 \mathrm{~h}$. The principal investigator subsequently reports a SAE to the Data Safety Monitoring Board (DSMB). This is an independent committee of trial experts that will focus on safety monitoring to ensure that the study meets the highest standards of ethics and patient safety.

\section{Data collection}

An overall schedule of trial-related activities and data collection of this study is shown in Table 1. All data will be collected on standardized CRFs designed for this study.

Baseline characteristics are collected including demographics, medical history, previous medication, pre-stroke mRS, vital signs, GCS score, NIHSS score, Diagnostic Scale of Syndrome Elements in Ischemic Stroke (DSSEIS) score, time of symptom onset, time of ED arrival, time of intervention, stroke subtypes, and complications.

Independent assessors at each center, who were trained before enrollment, will be responsible for assessment including NIHSS score, GCS score, DSSEIS score, and PRO score at $48 \mathrm{~h}, 7$ days, and 10 days or at discharge if earlier.

In addition, CD-ROM copies of all computed tomography (CT) and magnetic resonance imaging (MRI) scans will be collected from participating centers and assessed blindly by independent neuro-radiologists at the central trial imaging analysis center at the Institute for Cerebrovascular Disease of Beijing University of Chinese Medicine (ICVD).

\section{Follow-up}

Independent interviewers will contact all subjects by telephone and collect follow-up information using standard Telephone Interview Forms (TIFs) at 30 days and 3 months after stroke onset. The TIF is a standard questionnaire including structured $\mathrm{mRS}, \mathrm{BI}$, locations, stroke recurrences, new-onset vascular events, self-reported information on medication use, and rehabilitation at follow-up period. All telephone interviewers, with a neurological background, will be trained to contact participants according to a standard operating procedure (SOP) before trial recruitment. All interviews will be recorded and retained.

\section{Data management and quality assurance}

To ensure the accuracy and reliability of the data, an independent organization will be responsible for the data management. After the first participant has completed the study at each site, a site visit will be performed by the trial monitor. The monitor will review the CRFs and compare to the patient's source medical record. Any inadequacies or errors will be reviewed with the local coinvestigator and coordinator. Subsequently, site monitoring 
Table 1 Schedule of trial-related activities

\begin{tabular}{|c|c|c|c|c|c|c|}
\hline & & & reatment & & Follow & \\
\hline & Baseline & 48 hours & 7 days & $\begin{array}{c}10 \text { days or } \\
\text { at discharge }\end{array}$ & 30 days & 3 months \\
\hline Eligibility check & $\bar{x}$ & & & & & \\
\hline Demographics & $x$ & & & & & \\
\hline Medical history & $x$ & & & & & \\
\hline Vital signs & $x$ & & & $x$ & & \\
\hline Physical examination & $x$ & & & $x$ & & \\
\hline Neurological evaluation & $x$ & & & $x$ & & \\
\hline Previous medication & $x$ & & & & & \\
\hline $\mathrm{CT} / \mathrm{MRI}$ & $x$ & & & & & \\
\hline ECG & $\times$ & & & $x$ & & \\
\hline Laboratory examination & $x$ & & & $x$ & & \\
\hline Stroke subtypes & $x$ & & & & & \\
\hline Informed consent & $x$ & & & & & \\
\hline Allocation & $x$ & & & & & \\
\hline Intervention & $\leftarrow$ & & & & & \\
\hline Assessments & & & & & & \\
\hline NIHSS & $x$ & $x$ & $x$ & $x$ & & \\
\hline GCS & $x$ & $x$ & $x$ & $x$ & & \\
\hline $\mathrm{mRS}$ & & & & & $x$ & $x$ \\
\hline Barthel Index & & & & & $x$ & $x$ \\
\hline PRO & & & & $x$ & & \\
\hline TCM Syndrome & $x$ & $x$ & $\times$ & $x$ & & \\
\hline sICH & & & & $x$ & & $x$ \\
\hline AEs and SAEs & & & & $x$ & & $x$ \\
\hline $\begin{array}{l}\text { New-onset major } \\
\text { vascular events }\end{array}$ & & & & $x$ & & $x$ \\
\hline Overall mortality & & & & $x$ & & $x$ \\
\hline Concomitant medications & & & & $x$ & & $x$ \\
\hline Complications & $x$ & $x$ & $x$ & $x$ & & \\
\hline
\end{tabular}

Abbreviations: CT computed tomography scan, MRI magnetic resonance imaging, ECG electrocardiogram, NIHSS National Institutes of Health Stroke Scale, GCS Glasgow Coma Scale, mRS modified Rankin Scale, PRO Patient-reported Outcome Scale of Stroke, TCM traditional Chinese medicine, s/CH symptomatic intracranial hemorrhage, $A E s$ adverse events, SAEs serious adverse events

visits will be performed after every six participants enrolled at that site.

Data entry from the CRFs and TIFs will be performed in the web-based system by trained data-entry personnel using independent, dual data entry. Data are entered twice with each entry performed by a different personnel. Dataentry operators are trained to enter exactly what is recorded on the CRFs. Every structured field that is entered into the database has either a range check or internal consistency check, or both. Data-quality checks are performed by the clinical data system after the independent, dual data entry. All discrepant entries will be marked and recorded for resolution at the clinical data system. Subsequently, a trial monitor will review the two entries, resolve discrepancies, and query the entry operators and local coinvestigator if necessary. All discrepancies are manually reviewed.

All study-related data and documentation will be retained by the Data Management Center at the Institute for Cerebrovascular Disease of Beijing University of Chinese Medicine (ICVD).

Several approaches were used to coordinate the different centers in order to avoid the potential heterogeneity of data. Firstly, investigators from all centers were trained to understand the clinical trial protocol and were required to comply with the standard procedure through centralized and site training before recruitment. Secondly, all assessors at each center and telephone interviewers were trained before enrollment to assure consistency among assessors. Thirdly, an independent monitoring team will be responsible for reviewing the CRFs and make sure that they comply with the medical record.

\section{Sample size}

Based on the Third International Stroke Trial (IST-3), it is expected that $35 \%$ of the patients with AIS treated with usual care will have an independent outcome (mRS 0-2) [16]. According to previous studies [17-19], the absolute increase in the proportion of patients independent varied from 12 to $27 \%$. Conservatively, a $12 \%$ absolute increase in the intervention group is assumed, compared with the control group. With a power of $85 \%$ and a twosided test of a 5\% type I error, a sample size of 594 would be needed. Considering the dropout rate, approximately 720 participants will be enrolled in this study. 


\section{Statistical analyses}

Statistical analysis will be executed on the intention-totreat principle. All statistical tests will be performed at the 0.05 level of significance, unless otherwise specified. The Statistical Analysis System (Version 9.2, SAS Institute Inc., Cary, NC, USA) will be used to perform the statistical analyses.

Baseline characteristics will be summarized by means of simple descriptive statistics and be compared between the two treatment groups to assess covariate balance. The two-sample Student's $t$ test will be used for continuous variables and the chi-square test or Wilcoxon test will be used for categorical variables.

The primary analysis will be a comparison of the proportion of patients in each group who are independent (mRS 0,1 , and 2) at 3 months, for all those allocated XNJ versus all those allocated control using Pearson's chi-squared test. Additional analysis will performed using the generalized Cochran-Mantel-Haenszel (CMH) test statistic to compare the distribution of mRS scores between groups.

Secondary analyses include the reduction of NIHSS, the total score and score in four dimensions of PRO, incidence of cardiovascular events, mortality, the proportion of BI scores $\geq 90$, END, and sICH. Secondary outcome analyses will be carried out according to standard statistical principles for comparison of parametric or nonparametric distributions as appropriate. Multivariable regression analysis will be used to adjust for chance imbalances in the main prognostic variables between groups, such as age, stroke severity (NIHSS score), time since onset, ischemic stroke subtype, whether atrial fibrillation, and whether diabetes mellitus.

Pre-defined subgroup analyses will be performed of the effect of treatment at 3 months, subdivided by the following baseline features: time since onset, age, gender, clinical stroke syndrome using the Trial of Org 10172 in Acute Stroke Treatment (TOAST) classification, stroke severity according to the NIHSS, blood pressure at randomization, and TCM syndrome (Zheng, a key concept in TCM) [20].

\section{Missing data}

Missing data may cause seriously compromised inferences to scientific credibility of causal conclusions from clinical trials. Missing data are mainly caused by discontinued treatment due to adverse events, lack of tolerability, lack of efficacy, or simple inconvenience. To limit the extent of missing data, several approaches are taken according to previous experience [21]. When the assigned treatment is discontinued, efforts will be made to obtain the participant's consent for the collection of data on treatments and outcomes. Patients with no data available after randomization will have worst-case values assigned for the 3-month data point (e.g., $\mathrm{mRS}=6, \mathrm{BI}=0$ ).

\section{Discussion}

In the XMAS study with a multicenter PROBE design, placebo is not used as the control group. A doubleblind, placebo-controlled designed trial might be optimal; however, this design would lead to considerably higher costs as compared with the PROBE design. A potential advantage of the PROBE design is that the effect of $\mathrm{XNJ}$ in our trial will resemble the effect in clinical practice. The PROBE design has been widely discussed and demonstrated to yield the same result as a placebocontrolled design $[22,23]$. Moreover, the PROBE design has been performed in several previous studies of stroke intervention [16, 24].

Our study has several limitations. First, since this study is undertaken in China, it is uncertain whether the effects of XNJ would be similar in other ethnic groups. Moreover, due to the relatively short follow-up period of 3 months, it will remain unknown whether XNJ can reduce vascular events and mortality over longer periods.

In summary, the aim of the XMAS study is to determine whether the addition of $\mathrm{XNJ}$ to guideline-based standard care increases the proportion of patients independent at 3 months after AIS. If positive, this study will provide critical evidence for $\mathrm{XNJ}$, a traditional Chinese patent medicine, as a complementary approach that can be initiated after reperfusion therapy or when the AIS is not eligible for thrombolytic treatment.

\section{Trial status}

Recruitment to the study started in June 2016. The trial is currently on going.

\section{Additional file}

Additional file 1: The SPIRIT Checklist. (DOC $127 \mathrm{~kb}$ )

\section{Abbreviations}

AEs: Adverse events; AIS: Acute ischemic stroke; ALT: Alanine aminotransferase; AST: Aspartate aminotransferase; BI: Barthel Index; CFDA: China Food and Drug Administration; CM: Chinese medicine; CRFs: Case Report Forms; CT: Computed tomography; DALYs: Disabilityadjusted life years; DSMB: Data Safety and Monitoring Board; DSSEIS: Diagnostic Scale of Syndrome Elements in Ischemic Stroke; ED: Emergency department; END: Early neurological deterioration; GCS: Glasgow Coma Scale; ICH: Intracranial hemorrhage; ICVD: Institute for Cerebrovascular Disease of Beijing University of Chinese Medicine; IRB: Institutional Review Board; IST-3: The Third International Stroke Trial; ITT: Intention-to-treat; MRI: Magnetic resonance imaging; mRS: modified Rankin Scale; NIHSS: National Institutes of Health Stroke Scale; OCSP: Oxfordshire Community Stroke Project; PRO: Patient-reported outcome; PROBE: Prospective, randomized controlled, open-label trial with blinded endpoints assessment; RCT: Randomized controlled trial; rt-

PA: Recombinant tissue plasminogen activator; SAEs: Serious adverse events; TCM: Traditional Chinese medicine; TIFs: Telephone Interview Forms;

TOAST: Trial of Org 10172 in Acute Stroke Treatment; XNJ: Xingnaojing injection

\section{Acknowledgements}

We would like to thank all patients who have agreed to participate in the study, and to the many staff in the participating hospitals, without whom 
the study would not be possible. We would like to thank the Jiminkexin Group, who provide the Xingnaojing injection. We would also like to thank Professor Chenchen Wang and Professor David Kent from Tufts Medical Center (USA) for their great help and efforts on improving this protocol.

\section{Funding}

The XMAS trial was funded by The National Key Technology R\&D Program, with grant number 2014BAI10B05. This trial has been designed, conducted, analyzed, interpreted, and reported independently of the funding agencies.

\section{Availability of data and materials}

The authors declare that all relevant data will be included in the article or supplementary files. Additional data are available from the corresponding author on reasonable request.

\section{Authors' contributions}

$X X L$ wrote the drafts and the final manuscript. YG is the principal investigator and conceived the study, designed the protocol, applied for financial support and commented on all drafts and the final manuscript. KGC and LBK helped conceive the study and design the protocol and commented on the final manuscript. QL helped revise the statistical analysis plan and comment on the final manuscript. All authors have read and approved the final manuscript.

\section{Ethics approval and consent to participate}

The XMAS trial will be conducted according to the principles of the Declaration of Helsinki, as amended by the World Medical Association General Assembly in October 2013. Trial organization, data management, monitoring and reporting of the study will also be performed in accordance with the guidelines for Good Clinical Practice and other regulations. Patients will be given full and adequate oral and written information about the nature and purpose of the study, potential benefits and risks before allocation. The consent provider will be the patient if they are competent, or a legally authorized representative if the patient is not competent. This trial has been approved by the Institutional Review Board (IRB) of Dongzhimen Hospital affiliated to Beijing University of Chinese Medicine (Approval number. ECPJ-BDY-2015-02-01) as well as the local IRB of each site if required before the start of recruitment.

\section{Consent for publication}

Not applicable

\section{Competing interests}

The authors declare that they have no competing interests.

\section{Publisher's Note}

Springer Nature remains neutral with regard to jurisdictional claims in published maps and institutional affiliations.

\section{Author details \\ ${ }^{1}$ Department of Neurology, Dongzhimen Hospital affiliated to Beijing University of Chinese Medicine, 5 Haiyuncang, Dongcheng District, Beijing 100029, China. ${ }^{2}$ Institute for Cerebrovascular Disease of Beijing University of Chinese Medicine, 5 Haiyuncang, Dongcheng District, Beijing 100029, China. ${ }^{3}$ Department of Intensive Care Unit, Dongzhimen Hospital affiliated to Beijing University of Chinese Medicine, 5 Haiyuncang, Dongcheng District, Beijing 100029, China. ${ }^{4}$ Center for Evidence-based Medicine, the Word Federation of Chinese Medicine Societies, 19 Xiaoying Road, Chaoyang District, Beijing 100101, China.}

Received: 6 March 2017 Accepted: 28 September 2017 Published online: 16 October 2017

\section{References}

1. GBD 2013 Mortality and Causes of Death Collaborators. Global, regional, and national age-sex specific all-cause and cause-specific mortality for 240 causes of death, 1990-2013: a systematic analysis for the Global Burden of Disease Study 2013. Lancet. 2015;385(9963):117-71.

2. Krishnamurthi RV, Feigin VL, Forouzanfar MH, Mensah GA, Connor M, Bennett DA, et al. Global and regional burden of first-ever ischaemic and haemorrhagic stroke during 1990-2010: findings from the Global Burden of Disease Study 2010. Lancet Glob Health. 2013;1(5):e259-81.
3. Wang W, Jiang B, Sun H, Ru X, Sun D, Wang L, et al. Prevalence, incidence and mortality of stroke in China: results from a nationwide populationbased survey of 480,687 adults. Circulation. 2017;135(8):759-71.

4. Powers WJ, Derdeyn CP, Biller J, Coffey CS, Hoh BL, Jauch EC, et al. 2015 American Heart Association/American Stroke Association focused update of the 2013 guidelines for the early management of patients with acute ischemic stroke regarding endovascular treatment: a guideline for healthcare professionals from the American Heart Association/American Stroke Association. Stroke. 2015;46(10):3020-35.

5. Liu L, Wang D, Wong KSL, Wang Y. Stroke and stroke care in China. Stroke. 2011;42(12):3651-4

6. Wu B, Liu M, Liu H, Li W, Tan S, Zhang S, Fang Y. Meta-analysis of traditional Chinese patent medicine for ischemic stroke. Stroke. 2007;38(6):1973-9.

7. Yu L, Wang N, Zhang Y, Wang Y, Li J, Wu Q, Liu Y. Neuroprotective effect of muscone on glutamate-induced apoptosis in PC12 cells via antioxidant and $\mathrm{Ca}^{2+}$ antagonism. Neurochem Int. 2014;70:10-21.

8. Wei G, Huang Y, Li F, Zeng F, Li Y, Deng R, et al. Xing Nao Jing, prescription of traditional Chinese medicine, prevents autophagy in experimental stroke by repressing p53-DRAM pathway. BMC Complement Altern Med. 2015;15:377.

9. Wei J, Yao L, Yang L, Zhao W, Shi S, Cai Q, et al. Alteration of glutamate/ GABA balance during acute alcohol intoxication in rats: effect of Xingnaojing injection. J Ethnopharmacol. 2015;166:333-9.

10. Xia X, Li Q, Liu M. Neuroprotective effect of a formula, Moschus combined with Borneolum Synthcticum, from Traditional Chinese Medicine on ischemia stroke in rats. Evid Based Complement Alternat Med. 2014;2014:1-10.

11. Peng W, Yang J, Wang Y, Wang W, Xu J, Wang L, Xing Z. Systematic review and meta-analysis of randomized controlled trials of Xingnaojing treatment for stroke. Evid Based Complement Alternat Med. 2014;2014:210851.

12. van Swieten JC, Koudstaal PJ, Visser MC, Schouten HJ, van Gijn J. Interobserver agreement for the assessment of handicap in stroke patients. Stroke. 1988;19(5):604-7.

13. Brott T, Adams HJ, Olinger CP, Marler JR, Barsan WG, Biller J, et al. Measurements of acute cerebral infarction: a clinical examination scale. Stroke. 1989;20(7):864-70.

14. Wang X, Liu Q, Zhong H, Gao Y. Second run clinical verification of patientreported outcome scale of stroke. Chin J Tradit Chin Med Pharm. 2012; 27(05):1245-8 [Article in Chinese].

15. Mahoney Fl, Barthel DW. Functional evaluation: the Barthel Index. Md State Med J. 1965;14:61-5.

16. Sandercock P, Wardlaw JM, Lindley Rl, Dennis M, Cohen G, Murray G, et al. The benefits and harms of intravenous thrombolysis with recombinant tissue plasminogen activator within $6 \mathrm{~h}$ of acute ischaemic stroke (the third international stroke trial [IST-3]): a randomised controlled trial. Lancet. 2012; 379(9834):2352-63.

17. Gu C, Fu Y, Wang Y, Wu J. Xinnaojing injection in the treatment of acute ischemic stroke: a clinical trial of 85 patients. J Emerg Tradit Chin Med. 2009; 18(12):1989-90 [Article in Chinese].

18. Lian $\mathrm{Q}$, Dong H, 03. Clinical observation of Xinnaojing injection in the treatment of acute ischemic stroke. J Emerg Tradit Chin Med. 2010;19:430-1 [Article in Chinese].

19. Li M. Clinical efficacy of Xingnaojing injection in the treatment of acute ischemic stroke. Strait Pharm J. 2011;3(07):153-5 [Article in Chinese].

20. Su S, Lu A, Li S, Jia W. Evidence-based ZHENG: a traditional Chinese medicine syndrome 2013. Evid Based Complement Alternat Med. 2014;2014:1-2.

21. Little RJ, D'Agostino R, Cohen ML, Dickersin K, Emerson SS, Farrar JT, et al. The prevention and treatment of missing data in clinical trials. N Engl J Med. 2012;367(14):1355-60.

22. Smith DH, Neutel JM, Lacourciere Y, Kempthorne-Rawson J. Prospective, randomized, open-label, blinded-endpoint (PROBE) designed trials yield the same results as double-blind, placebo-controlled trials with respect to ABPM measurements. J Hypertens. 2003;21(7):1291-8.

23. O'Neil WM, Welner SA, Lip GY. Do open label blinded outcome studies of novel anticoagulants versus warfarin have equivalent validity to those carried out under double-blind conditions? Thromb Haemost. 2013;109(3): 497-503.

24. Westendorp WF, Vermeij J, Zock E, Hooijenga IJ, Kruyt ND, Bosboom HJLW, et al. The Preventive Antibiotics in Stroke Study (PASS): a pragmatic randomised open-label masked endpoint clinical trial. Lancet. 2015; 385(9977):1519-26. 